\title{
Recurrent allograft C3 glomerulonephritis and unsuccessful eculizumab treatment
}

\section{Kaartinen, Kati}

2018-02

Kaartinen , K, Martola , L , Räisänen-Sokolowski , A \& Meri , S 2018 , ' Recurrent allograft C3 glomerulonephritis and unsuccessful eculizumab treatment ' , Clinical Immunology, vol. 187 , pp. 104-106 . https://doi.org/10.1016/j.clim.2017.10.013

http://hdl.handle.net/10138/301307

https://doi.org/10.1016/j.clim.2017.10.013

publishedVersion

Downloaded from Helda, University of Helsinki institutional repository.

This is an electronic reprint of the original article.

This reprint may differ from the original in pagination and typographic detail.

Please cite the original version. 


\title{
Recurrent allograft C3 glomerulonephritis and unsuccessful eculizumab treatment
}

\author{
Kati Kaartinen $^{\text {a,* }}$, Leena Martola ${ }^{a}$, Anne Räisänen-Sokolowski ${ }^{\text {b }}$, Seppo Meri ${ }^{\text {c,d }}$ \\ a Department of Nephrology, Abdominal Center, Helsinki University Central Hospital, Helsinki, Finland \\ b Transplantation Laboratory, HUSLAB, Department of Pathology. Helsinki University Central Hospital, Helsinki, Finland \\ c Department of Bacteriology and Immunology, Immunobiology Research Program, Helsinki University, Helsinki, Finland \\ d HUSLAB, Helsinki University Central Hospital, Helsinki, Finland
}

\section{A R T I C L E I N F O}

\section{Article history:}

Received 28 March 2017

Received in revised form 25 September 2017

Accepted with revision 28 October 2017

Available online 31 October 2017

\section{Keywords:}

C3 glomerulonephritis

Transplantation

C3 nephritic factor

Eculizumab

\begin{abstract}
A B S T R A C T
There is a great lack of efficient treatments for membranoproliferative glomerulonephritis (MPGN) and recently emerged complement therapies have been proposed to be useful. We report a patient with a complementmediated MPGN having recurrencies in kidney allografts and an unsuccessful treatment with complement inhibitor, eculizumab (anti-C5 monoclonal antibody). Nephritic factor (C3Nef), an autoantibody against C3bBb, in the patient serum activated C3 but not C5 showing that major damage was mediated by C3 activation with clearly less involvement of $\mathrm{C} 5$ explaining unresponsiveness to eculizumab. Analyzing C3Nef-mediated C 3 and C5 activation separately could help in choosing the right patients for eculizumab therapy.
\end{abstract}

(c) 2017 Elsevier Inc. All rights reserved.

\section{Introduction}

Understanding the etiology of MPGN has gone through a major shift leading also to a new classification in recent years. The newest classification relying on immunofluorescence microscopy (IF) divides it into two subtypes: complement-mediated ( $\mathrm{C} 3$ and no or very little immunoglobulins) and immune-complex-mediated (IF showing both C3 and immunoglobulins) [1]. The former will arise following dysregulation of the alternative pathway (AP) of complement and the latter as a result of ongoing antigenemia due to chronic infection, paraproteinemia, autoimmune disease or miscellaneous reasons. C3 glomerulopathies (C3G) refer to complement-mediated MPGN that can be further subclassified into dense deposit disease (DDD) and C3 glomerulonephritis (C3GN) by electron microscopy (EM). Both DDD and C3GN are rare diseases of dysregulation of the alternative pathway. They are either due to mutations in complement components or autoantibodies against $\mathrm{C} 3 \mathrm{bBb}$ (C3Nef) or factor H or rarely factor B [2]. Due to strong AP activation the levels of complement activation products are elevated and $\mathrm{C} 3$ is consumed in the plasmas of C3G patients [3]. C3Nef is detected in the majority of patients with DDD and approximately in half of the patients with C3GN $[4,5]$.

Currently, there is no therapeutic agent of proven efficacy for C3G. Supportive measures, occasional trials of plasma therapy and immune suppression have been reported with varying results [6]. Preliminary

\footnotetext{
* Corresponding author at: Pietarinkatu 2 C 25, 00140 Helsinki, Finland.

E-mail address: kati.kaartinen@hus.fi (K. Kaartinen).
}

results using the complement inhibitor, eculizumab, were promising, especially in cases with elevated blood levels of SC5b-9, the soluble form of the terminal complement complex. Improvements in renal variables were seen in about $60 \%$ of patients, and the results did not seem to depend on the type of C3G [7-11]. However, upstream activation at the C3 level continues even though C5 and downstream terminal pathway activation are completely blocked with eculizumab. Extensive C3 activation could thereby cause substantial progression of the disease $[3,12]$. It would be important to differentiate C3G patients likely to respond to eculizumab from those not responding without a treatment trial with off-label use of expensive medication.

\section{Methods}

Complement analyses were carried out at HUSLAB, an accredited laboratory of the Helsinki University Hospital. Complement component levels (C3, C4) were determined by nephelometry. The Wielisa ${ }^{\circledR}$ test was used for determination of activities of the classical, lectin and alternative complement pathways (Euro-Diagnostica, Malmö, Sweden). Plasma soluble terminal complement complex (p-SC5b-9) was analyzed at the Department of Medical Microbiology and Immunology, Turku University by a sandwich-ELISA-test with a murine monoclonal anti-human SC5b-9 (Quidel) as a capture-antibody. The SC5b-9 complex is recognized by polyclonal $\mathrm{C} 5$ and $\mathrm{C} 7$ antibodies and detected by a HRP-conjugated secondary antibody. Complement gene mutation analyses were performed by Secugen S.L. in Madrid, Spain. Factor H antibodies were analyzed by an in-house ELISA technique and factor $\mathrm{H}$ - 
related proteins 1-5 (FHR-1-5) were analyzed by an immunoblotting technique using a polyspecific anti-human CFH antibody at the Department of Bacteriology and Immunology, University of Helsinki. C3Nef analysis was performed by incubating patient serum with fresh normal human serum in veronal-buffered saline (VBS) or $5 \mathrm{mM} \mathrm{MgCl} 2,10 \mathrm{mM}$ EGTA-VBS or in $10 \mathrm{mM}$ EDTA-VBS at $37^{\circ} \mathrm{C}$ for $30 \mathrm{~min}$. Subsequent C3 and $\mathrm{C} 5$ cleavage were analyzed by immunoblotting after electrophoresis in agarose or SDS-PAGE gels using rabbit antibodies against human C3c or C5 from Quidel.

Kidney histology was examined at the Transplantation Laboratory, Department of Pathology, Helsinki University Hospital. Hematoxylin and eosin (HE), Periodic Acid Schiff (PAS) and Jones Silver stainings were used for LM. Deposition of complement components C1q, C4d, C3 and immunoglobulins ( $\operatorname{IgG}$, IgM) in cryostat kidney sections were analyzed by indirect IF microscopy.

\section{Case report and results}

A male patient, currently 37 years old, was diagnosed with MPGN type I already at the age of 16 in 1993. Kidney function was lost in the coming years and hemodialysis was started in 1997. The patient received the 1st cadaveric kidney transplantation in 1998, with conventional triple immunosuppression (cyclosporine, azathioprine and corticosteroids) and an immediate good graft function. Several kidney biopsies were performed in the coming years and a clear deposition of $\mathrm{C} 3$ was observed in the mesangial areas in 2002. Hemodialysis was restarted in 2003. The 2 nd cadaveric transplantation was performed in 2006 with tacrolimus, mycophenolate mofetil and corticosteroids based immunosuppression with an immediate good graft function. Protocol biopsy at 3 months showed weak IgM and a clear C3 staining in glomeruli. Allograft function was normal, but both hematuria and proteinuria were increasingly evident. Another biopsy at 12 months showed clear signs of MPGN with strong glomerular staining for C3 and some IgM. Allograft dysfunction developed along with nephrotic range proteinuria and hematuria. Oral cyclophosphamide was started for 2 months, plasma exchanges were instituted, tacrolimus and corticosteroids were maintained and mycophenolate mofetil was stopped. Due to a lack of response rituximab was started with no apparent response. Finally, home hemodialysis was started in 2009.

The 3rd cadaveric transplantation took place in April 2013, but the patient remained dependent on dialysis afterwards. Transplant baseline biopsy was normal. The 2 nd biopsy 18 days posttransplantation showed acute tubular necrosis (ATN) in large areas. Glomerular endothelial basement membranes showed slight damage indicating MPGN recurrence. No IF analysis was performed. The 3rd biopsy at 25 days showed ATN in some areas and also clear MPGN with C3 in the capillaries and mesangium with no immunoglobulins, C1q or C4d. Several transplant biopsies were taken during the coming months all showing increasing MPGN changes and $\mathrm{C} 3$ in the glomeruli. Eventually ATN vanished, and no signs of rejection or inflammation were detected. In the last biopsy all glomeruli showed typical features of MPGN, but no significant atrophy, fibrosis or inflammation was present. C4d staining was negative, C3 was strongly positive in the glomerular capillaries and mesangium. EM showed extensive glomerular damage, but the podocytes were reasonably well preserved. On the basis of EM the diagnosis of C3 glomerulonephritis was confirmed.

Complement inhibitor eculizumab, a humanized anti-C5 antibody (Soliris ${ }^{\circledR}$, Alexion, Cheshire, USA), was started in the beginning of July 2013, nine weeks after the transplantation with a protocol similar to that used in atypical hemolytic-uremic syndrome (aHUS). The patient remained dialysis-dependent all the time, but the frequency was reduced to twice weekly one month after starting the eculizumab treatment. Triple immunosuppression (corticosteroids, mycophenolate mofetil and tacrolimus) was continued during eculizumab therapy. Predialysis serum creatinine was $4.1 \mathrm{mg} / \mathrm{dl}$, daily urinary protein excretion $315 \mathrm{mg} / 24 \mathrm{~h}$ and urine dipstick showed hematuria at the time of starting eculizumab treatment. Predialysis serum creatinine was at its lowest varying between 3.7 and $3.9 \mathrm{mg} / \mathrm{dl}$ three months after starting eculizumab. However, a disturbed acid-base balance necessitated medication between the dialysis sessions. Three times a week schedule was restarted after approximately 5 months of eculizumab treatment due to progressive deterioration of the fluid status and increasing creatinine levels. Eculizumab was discontinued after 6 months due to a poor response. Currently, the patient is performing home hemodialysis $4 \mathrm{~h}$ every other day.

Complement investigations before the start of complement inhibitor therapy showed increased levels of p-SC5b-9 $3525 \mathrm{ng} / \mathrm{ml}$ ( $\mathrm{ref}<725 \mathrm{ng} / \mathrm{ml}$ ). Total complement activities of the classical, lectin and alternative pathways were within the reference range, but the level of plasma C3 was decreased (0.69 g/l; ref. 0.71-1.41), plasma $\mathrm{C} 4$ was normal and $\mathrm{C} 3 \mathrm{Nef}$ was constantly positive. Sequence analysis of complement factor $\mathrm{H}$ ( $\mathrm{CFH})$, factor I ( $\mathrm{CFI}$ ), membrane cofactor protein (MCP, CD46), factor B (CFB), C3, diacylglycerol kinase $\varepsilon$ (DGKE) and thrombomodulin (THBD) genes revealed no mutations. However, in the MCP gene, a heterozygous aHUS risk haplotype and several risk polymorphisms in CFB and C3 and one known polymorphism in THBD were observed, but they were not considered significant. No deficiencies of FHR-1-5 proteins or anti-factor $\mathrm{H}$ antibodies were detected. We next analyzed complement activation by mixing and incubating ( $30 \mathrm{~min}$ at $37^{\circ} \mathrm{C}$ ) the patient's serum (taken when not under eculizumab treatment) with normal serum from a healthy volunteer. Analysis by SDS-PAGE and immunoblotting with specific polyclonal antisera showed that $\mathrm{C} 3$ became activated, but $\mathrm{C} 5$ not in the serum mixture. The result suggests that $\mathrm{C} 3 \mathrm{Nef}$ in the patient was of the type that in solution activates $\mathrm{C} 3$ but not C5.

Eculizumab blocked activities of all the complement activation pathways and reduced p-SC5b-9-levels significantly. C3Nef remained constantly positive and plasma $\mathrm{C} 3$ level never increased to normal levels. This was as expected considering that eculizumab acts at the C5 level in the complement cascade. A series of light microscopy (LM) and IF analyses of C3 clearly show the evolution of C3G/MPGN histology before and during eculizumab treatment (Figs. 1 and 2).

\section{Discussion}

Our patient seemed to benefit from eculizumab treatment during the first few months of therapy. Plasma level of p-SC5b-9 was initially increased and subsequently decreased after the start of eculizumab treatment and there was no major chronic damage seen in a kidney biopsy in the beginning raising the hope for a treatment response with eculizumab. However, repeated kidney biopsies showed a progressive MPGN-type glomerular injury and the clinical benefit was minimal. If eculizumab had been started earlier, the benefit could have been greater, but not necessarily substantial.

The patient was regularly positive for C3 nephritic factor, an autoantibody that stabilizes the AP C3 convertase C3bBb. We wanted to analyze whether continuous overactivation of $\mathrm{C} 3$ was the primary problem as complement C5-driven injury seemed to be efficiently blocked by eculizumab. Therefore, we carried out further complement analysis to determine whether $\mathrm{C} 5$ could have been involved in the complement-driven renal damage. When incubating the patient's serum with that of a healthy person a substantial C3 activation, but no $\mathrm{C} 5$ activation was observed. This was compatible with the course of the disease as eculizumab has no impact on complement activation before $\mathrm{C} 5$. This is in line with a similar finding as eculizumab added in vitro to serum of DDD patient did not 

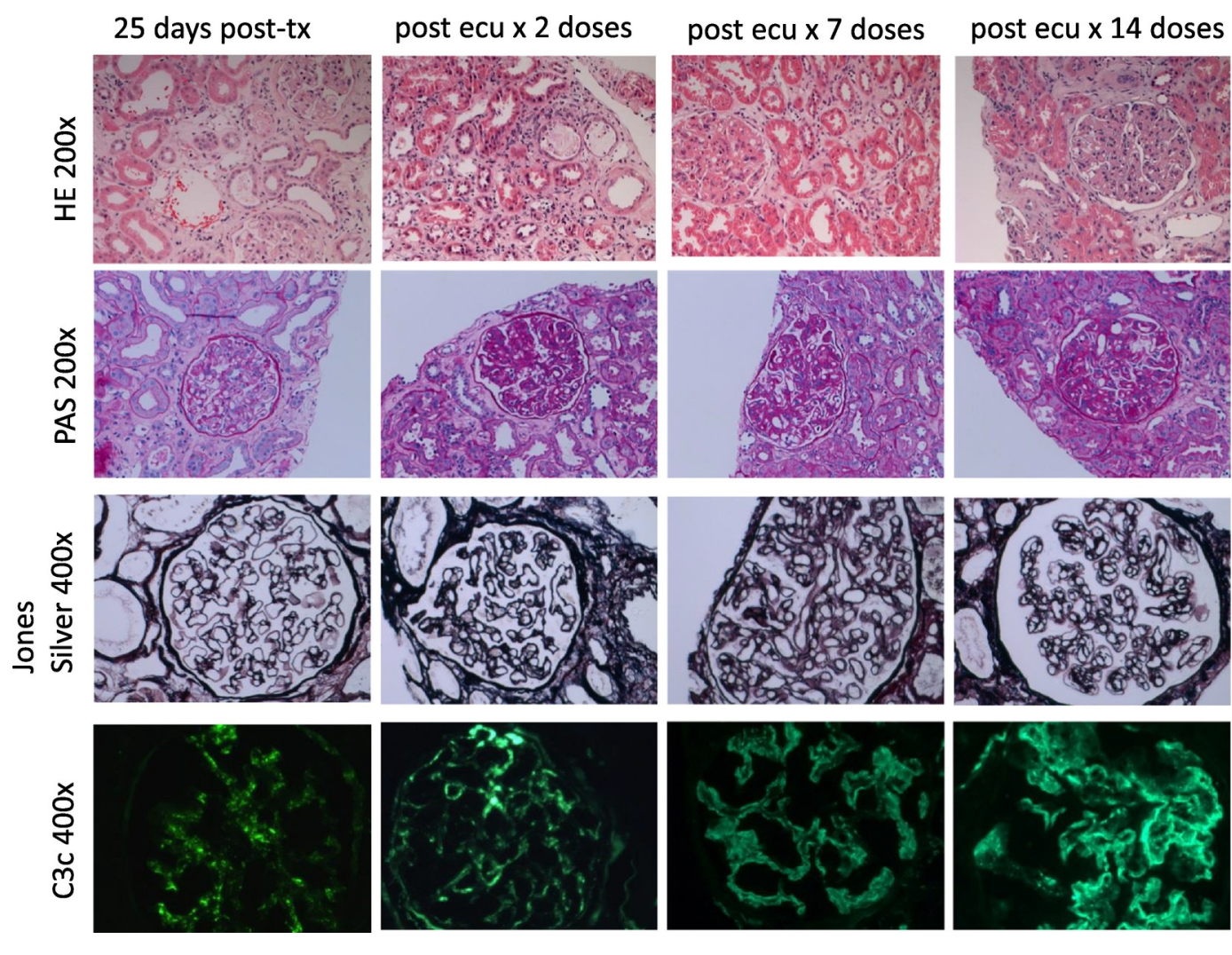

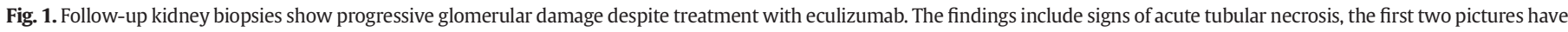

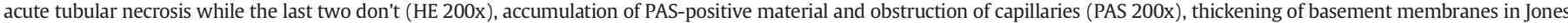

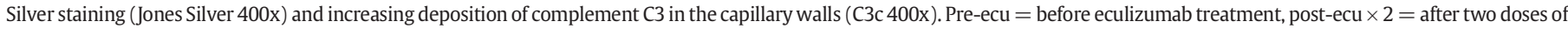
eculizumab, post-ecu $\times 7=$ after seven doses of eculizumab, post-ecu $\times 14=$ after 14 doses of eculizumab.

influence C3a generation, but effectively blocked C5a generation [13]. The fact that the plasma level of SC5b-9 was initially elevated in our patient could be due to the activity of the $\mathrm{C} 5$ convertase mostly as a cell surface enzyme complex.

The result suggests that $\mathrm{C} 3 \mathrm{Nef}$ in the patient was of the type that in solution activates $\mathrm{C} 3$ but not $\mathrm{C} 5$. We therefore propose that methods dissecting C 3 and $\mathrm{C} 5$ activation could be employed to assess which patients would be likely to benefit from the off-label use of eculizumab therapy without having to reach that conclusion after a treatment trial.

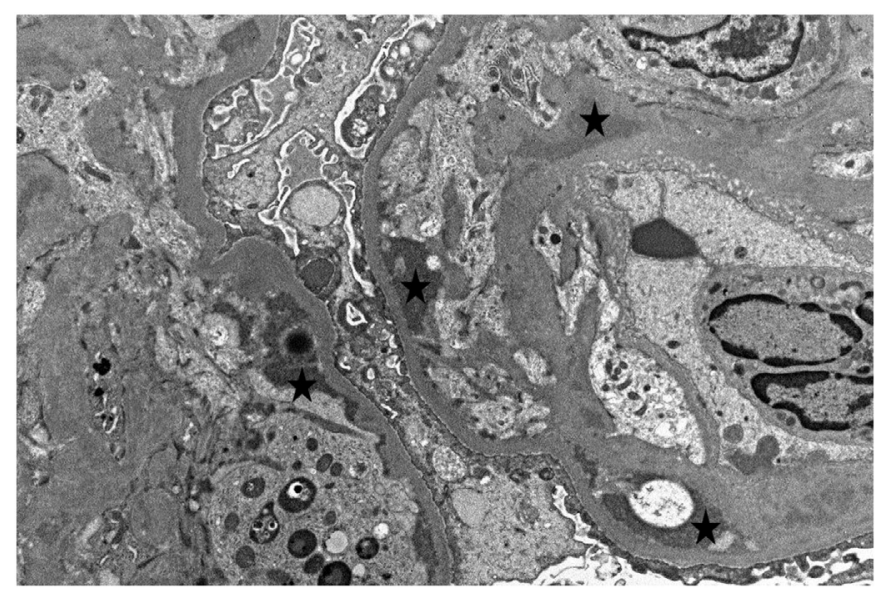

Fig. 2. Electronmicrograph demonstrating subendothelial deposits ( $\star$ ) in biopsy after 14 doses of Ecu.

\section{Disclosures}

Kati Kaartinen, Leena Martola and Seppo Meri have received consultancy fees from Alexion.

\section{References}

[1] S. Sethi, F. Fervenza, Membranoproliferative glomerulonephritis-a new look at an old entity, N. Engl. J. Med. 366 (2012) 1119-1131.

[2] A. Angioi, F. Fervenza, S. Sethi, et al., Diagnosis of complement alternative pathway disorders, Kidney Int. (2) (2016) 278-288.

[3] Y. Zhang, C. Nester, B. Martin, et al., Defining the complement biomarker profile of C3 glomerulopathy, Clin. J. Am. Soc. Nephrol. 9 (2014) 1876-1882.

[4] S. Sethi, F. Fervenza, Y. Zhang, et al., C3 glomerulonephritis: clinicopathological findings, complement abnormalities, glomerular proteomic profile, treatment, and follow-up, Kidney Int. (4) (2012) 465-473.

[5] A. Servais, L. Noël, L. Roumenina, et al., Acquired and genetic complement abnormalities play a critical role in dense deposit disease and other $\mathrm{C} 3$ glomerulopathies, Kidney Int. (4) (2012) 454-464.

[6] C.M. Nester, R.J. Smith, Treatment options for C3 glomerulopathy, Curr. Opin. Nephrol. Hypertens. 22 (2013) 231-237.

[7] J.A. McCaughan, D.M. O'Rourke, A.E. Courtney, Recurrent dense deposit disease after renal transplantation: an emerging role for complementary therapies, Am. J. Transplant. 12 (2012) 1046-1051.

[8] E. Daina, M. Noris, G. Remuzzi, Eculizumab in a patient with dense-deposit disease, N. Engl. J. Med. 366 (2012) 1161-1163.

[9] A. Bomback, R. Smith, G. Barile, et al., Eculizumab for dense deposit disease and C3 glomerulonephritis, Clin. J. Am. Soc. Nephrol. 7 (2012) 748-756.

[10] M. Vivarelli, A. Pasini, F. Emma, Eculizumab for the treatment of dense-deposit disease, N. Engl. J. Med. 366 (2012) 1163-1165.

[11] S. Radhakrishnan, A. Lunn, M. Kirschfink, et al., Eculizumab and refractory membranoproliferative glomerulonephritis, N. Engl. J. Med. 366 (2012) 1165-1166.

[12] S. Gurkan, B. Fyfe, L. Weiss, et al., Eculizumab and recurrent C3 glomerulonephritis, Pediatr. Nephrol. 28 (2013) 1975-1981.

[13] O. Chen, M. Wiesener, H. Eberhardt, et al., Complement factor H-related hybrid protein deregulates complement in dense deposit disease, J. Clin. Invest. (1) (2014) $145-155$. 